\title{
APLICACIONES DE LA SEMÁNTICA DE ESQUEMAS
}

\author{
Ana María Rojo López \\ Universidad de Murcia
}

\begin{abstract}
RESUMEN. La Semántica de Esquemas ('Frame Semantics') es una teoría sobre el significado iniciada por Charles Fillmore en los años 70 y que se ha seguido desarrollando hasta la actualidad. Aunque la Semántica de Esquemas se ha concentrado sobre todo en el estudio del significado a nivel léxico, cada vez son más los trabajos que intentan describir el papel de los esquemas en el análisis textual y discursivo. Este artículo presenta algunas de las aplicaciones concretas de esta teoría a ámbitos como la lexicografía, la comprensión lectora, la traducción y el análisis del discurso.
\end{abstract}

ABSTRACT. Frame Semantics' is a theory of meaning Charles Fillmore started in the 70 s and has continued to develop to the present day. Although Frame Semantics has mainly focused on the study of lexical meaning, we can find an increasing number of works that aim to describe the role frames play on textual and discourse analysis. This article presents some of the applications of this theory to fields such as lexicography, reading comprehension, translation and discourse analysis.

\section{Introducción}

En los años 70 Charles Fillmore inició una teoría sobre el significado denominada Semántica de Esquemas' ('Frame Semantics'). En su artículo 'An Alternative to Checklist Theories of Meaning' (1975), Fillmore presenta la Semántica de Esquemas como alternativa a las teorías semánticas que intentan captar el significado de un término lingüístico mediante una serie de rasgos o criterios que definen sus condiciones de verdad y actúan como condiciones suficientes y necesarias para su uso apropiado. Con la Semántica de Esquemas, Fillmore propone sustituir las listas de criterios por una teoría basada en las nociones de 'prototipo' (en el sentido desarrollado por Rosch ${ }^{2}$

1. 'Semántica de Esquemas' se ha elegido como traducción de 'Frame Semantics' por los siguientes motivos: por una parte, aunque es posible encontrar 'frame' traducido por 'marco', la traducción por 'esquema' parece resaltar de forma más clara el carácter estructurado de estos constructos. Además, el término 'esquema' parece ser la denominación genérica más común para estas estructuras en diversas teorías de representación del conocimiento (De Vega 1984; Sierra Díez 1994).

2. Rosch $(1973,1978)$ demostró que la categorización se basa en la idea del 'prototipo'; los seres humanos categorizamos nuestras experiencias en categorías prototípicas que más tarde utilizamos para 
en psicología cognitiva) y 'frame' (que toma del ámbito de la inteligencia artificial y la psicología cognitiva). En líneas muy generales, la idea básica es que existen determinados términos o expresiones lingüísticas que se asocian con ciertas situaciones prototípicas o 'esquemas' necesarios para interpretar correctamente el significado de dichas expresiones.

Con la Semántica de Esquemas, Fillmore pretende demostrar que el significado de una palabra no es una simple lista de atributos sino más bien una estructura conceptual compleja basada en la experiencia. Esta base 'experiencial' del significado nos ayuda a comprender ciertos aspectos de la naturaleza del lenguaje que no son explicables desde una semántica basada en listas de atributos; por ejemplo, nos ayuda a explicar por qué determinados usos nos resultan extraños o atípicos. Sin duda, todos conoceremos a alguna mujer ya entrada en años y soltera a la que alguna vez habremos calificado un tanto cruelmente de 'SOLTERONA'. Sin embargo, si esta señora fuera monja, el apelativo de 'solterona' nos resultaría extraño o incluso inapropiado. Las dos muestran los mismos atributos, es decir, ambas son mujeres, de una cierta edad y no están casadas. ¿Dónde está entonces la diferencia? Para explicar esta diferencia, necesitamos recurrir a un modelo cultural prototípico: ser monja es un caso atípico en una sociedad en la que las mujeres desean el matrimonio, se casan a una cierta edad, con un solo hombre y permanecen casadas hasta la muerte. Las mujeres que no están casadas cuando deberían estarlo se denominan 'solteronas'. Es más, el término se asocia a cierto sentimiento de fracaso o amargura causado al no haber conseguido el deseable estatus del matrimonio. Prueba del carácter cultural de este modelo prototípico es que cada vez se utiliza menos este término para designar a una mujer que no se ha casado: en la sociedad moderna el matrimonio está dejando de ser el estatus ideal de la mujer trabajadora, que cada vez tiene menos tiempo para la familia.

De la misma forma, la base experiencial del significado nos puede ayudar a comprender ciertos fenómenos de la adquisición del lenguaje que resultan difícilmente explicables desde la idea tradicional de la categorización como proceso basado en una lista de propiedades. En su artículo 'An Alternative to Checklist Theories of Meaning', Fillmore menciona la experiencia de Mary Erbaugh, del departamento de lingüística de Berkeley. Tras enseñar un pomelo a un grupo de niños, Erbaugh lo peló y lo cortó en gajos, comiéndoselo como si fuera una naranja. Sorprendentemente, aunque los niños habían reconocido en un principio que se trataba de un pomelo, la forma de comérselo de Erbaugh les indujo a pensar que se habían equivocado y que en realidad se trataba de una naranja. Para los niños, un pomelo era una fruta que se corta por

comprender otros conceptos. Desde esta perspectiva, la comprensión consiste en reconocer los modos en los que un objeto o concepto puede considerarse un ejemplo del inventario de prototipos que tenemos almacenados en nuestra memoria semántica. De esta forma, Rosch se aleja de la idea de categoría aristotélica, reemplazando la naturaleza binaria de esta categoría clásica (o se pertenece a la categoría o se está fuera de ella) por un continuo que permite distintos grados de pertenencia a una categoría. 
la mitad y se come con una cuchara. Por lo tanto, la fruta que se había comido Erbaugh no podía ser un pomelo; obviamente, aquello tenía que ser una naranja. La experiencia de Erbaugh muestra que los niños todavía no habían separado las categorías de 'pomelo' y 'naranja' de su propia experiencia de escenas de gente comiendo estas frutas.

Situaciones como la descrita por Fillmore ocurren continuamente en la vida cotidiana de un niño. Otro ejemplo que parece corroborar la base experiencial del significado podría ser el del niño que se empeñaba en llamar de la misma forma tanto a Mortadelo y Filemón como a Zipi y Zape o al mismísimo Rompetechos. Después de mucho pensar, su madre descubrió que el niño los incluía a todos en la misma categoría que al personaje televisivo 'Chavo', ${ }^{3}$ hasta entonces el único personaje tipo 'comic' que el niño conocía. Este hecho, que en un principio puede parecer trivial, sin embargo, demuestra que la categorización consiste en reconocer las formas en las que un objeto puede considerarse un ejemplo del inventario de prototipos que tenemos disponibles en nuestras mentes. Para este niño, los personajes del comic no eran más que ejemplos del prototipo de 'CHAVO', de la misma manera que para la mayoría de los niños todos los animales son 'perros' o 'guau-guaus'. De esta forma, la noción de 'prototipo' nos ayuda a explicar esta peculiar tendencia de los niños a 'generalizar' en exceso; sin embargo, resulta más difícil explicar dicho fenómeno basándonos en una lista de rasgos o propiedades.

En este trabajo argumentaremos precisamente a favor de la capacidad explicativa del 'esquema' tanto en cuestiones de semántica léxica como en el ámbito más complejo del análisis del discurso.

\section{Aplicaciones de la Semántica de Esquemas}

Una vez revisados los principios básicos de la Semántica de Esquemas, este apartado se dedica a aplicaciones concretas de esta teoría en ámbitos como la lexicografía, la interpretación textual, el análisis del discurso y la traducción.

\subsection{Semántica de Esquemas y lexicografía}

Sin duda alguna, la aplicación de la Semántica de Esquemas más explotada por el propio Fillmore ha tenido lugar en el ámbito de la lexicografía. La idea básica que sirve de punto de partida a este uso de la Semántica de Esquemas es que la utilización de esta teoría en el análisis léxico puede contribuir a mejorar la calidad de los diccionarios actuales.

3. El personaje de 'Chavo' es el protagonista de una serie infantil mejicana titulada 'El chavo del 8', que durante algún tiempo se ha estado mostrando en la segunda cadena de TVE. 
Entre las aplicaciones de la Semántica de Esquemas al análisis lexicográfico destacan Fillmore y Atkins (1992), Fillmore y Hirose (1992), Atkins (1994). Todos ellos tienen como objetivo proponer un análisis que solucione al menos algunos de los problemas planteados en los diccionarios. Estos problemas se reducen a los siguientes: desde un punto de vista semántico, la mayoría de los diccionarios no explican las diferencias entre términos semánticamente relacionados, dejando sin describir fenómenos como la homonimia, polisemia entre los distintos sentidos de una palabra y relaciones entre sinónimos. Desde el punto de vista de la información gramatical, a menudo los diccionarios no especifican los patrones sintácticos de los distintos términos o sentidos de un mismo término. Como consecuencia, la capacidad descriptiva de los diccionarios es bastante limitada y a menudo no es suficiente para explicar el uso de los términos en un corpus. Todos los trabajos mencionados intentan demostrar que un acercamiento basado en la Semántica de Esquemas puede contribuir a superar estas limitaciones de los diccionarios convencionales (tanto monolingües como bilingües), permitiéndonos acceso al esquema que subyace al significado de una palabra.

Todos los estudios utilizan una metodología bastante similar, aunque mientras que Fillmore \& Atkins (1992) y Atkins (1994) se centran en el análisis del inglés, el trabajo de Fillmore \& Hirose (1992) presenta ciertas diferencias, manejando un análisis comparado de dos lenguas distintas (inglés y japonés). Pero a pesar de estas diferencias, todos los trabajos utilizan un método semejante:

- Llevan a cabo una recogida de datos en forma de corpus.

- Especifican los distintos elementos que configuran el esquema.

- Clasifican los distintos sentidos o 'esquemas conceptuales' ('conceptual schemas') del término basándose en las diferencias en la configuración de elementos del esquema.

- Establecen la Relación entre los elementos del esquema que intervienen en los distintos sentidos y su realización sintáctica.

- Elaboran un análisis de la relación entre palabras asociadas.

Quizás el ejemplo más claro de este tipo de análisis sea el estudio que Fillmore y Atkins (1992) realizan del verbo 'risk', el cual exponemos brevemente a continuación.

\subsection{1. 'Starting where the dictionaries stop: the challenge of corpus lexico- graphy' (Fillmore \& Atkins 1994)}

Tras consultar las definiciones de 'risk' ofrecidas en diez diccionarios monolingües distintos, Fillmore y Atkins señalan la confusión existente no sólo en cuanto a la diferenciación entre los distintos sentidos del término, sino también en lo referente a los patrones sintácticos de complementación. Es más, las definiciones no consiguen distinguir de forma clara entre expresiones casi sinónimas como 'run a risk' y 'take a risk'. Las limitaciones del formato convencional ofrecido por los diccionarios quedan sobre todo patentes al aplicarlo al análisis de un corpus. Las definiciones de 'risk' 
ofrecidas por los diccionarios no pueden explicar un gran número de las apariciones del término contenidas en el corpus.

Para Fillmore y Atkins, el principal problema de las entradas de los diccionarios es que se limitan simplemente a diferenciar entre los sentidos de un término sin analizar el esquema que subyace a su significado. En el caso de 'risk' los elementos que conforman este esquema son los siguientes:

\begin{tabular}{|c|c|c|}
\hline Protagonista & {$[\mathbf{P r}]$} & La persona central del esquema \\
\hline Mal & [Ma] & El posible daño o resultado negativo \\
\hline Decisión & [De] & La decisión que lo provocaría \\
\hline Meta & [Me] & El objetivo o resultado deseado \\
\hline Situación & {$[\mathbf{S t}]$} & La situación en la que existe el riesgo \\
\hline Posesión & [Po] & $\begin{array}{l}\text { Algo o alguien que el protagonista valora y que es arriesgado } \\
\text { en la situación }\end{array}$ \\
\hline
\end{tabular}

Partiendo de esta estructura, identifican tres sentidos o 'escenarios' en los que se utiliza el término 'risk' y que resultan de una configuración distinta de los elementos del esquema:

1.En el primer 'escenario', el protagonista se enfrenta a un posible daño de manera involuntaria, sin llevar a cabo ninguna acción o decisión que le sitúe en esa situación de peligro. Este sentido estaría ejemplificado por la oración He risked falling down y la configuración de elementos sería [Pr, Ma].

2.En el segundo sentido, la acción del protagonista pone en peligro a algo o alguien. Un ejemplo de este escenario es la oración He risked his life, que presenta la configuración [Pr, Po].

3.Un tercer sentido es aquel en el que el protagonista toma una decisión, siendo consciente de las posibles consecuencias negativas de su acción; la configuración sería por lo tanto [Pr, De] y un ejemplo podría ser He risked climbing the mountain.

Fillmore y Atkins también señalan que cada uno de estos escenarios requiere una complementación sintáctica distinta:

- La posesión [Po] siempre aparece como un SN (I risked my life for you).

- El posible daño o mal resultado [Ma] puede aparecer, bien como un SN (we risked death to help you), bien como un SV + gerundio (we risked being killed).

- La decisión consciente del protagonista [De] también puede realizarse, bien como un SN (he risked a swim), bien como un SV + gerundio (he risked swimming in the river).

Este esquema nos permite explicar de forma explícita la ambigüedad de determinados casos suceptibles de una doble interpretación. Un ejemplo de uso ambiguo es el caso de la oración He risked a battle, la cual puede interpretarse como si a) el protagonista 
tomara la decisión consciente de pelear, escogiendo la configuración [Pr, De]; o como si b) se viera envuelto en la batalla sin ninguna acción o decisión consciente por su parte, en cuyo caso, la configuración de elementos del esquema sería [Pr, Ma]. Estas dos configuraciones sirven además para explicar la diferencia entre las expresiones 'run a risk' [Pr, Ma] y 'take a risk' [Pr, De]: mientras que 'take a risk' implica la decisión consciente del protagonista, 'run the risk' no conlleva necesariamente una decisión consciente.

Aunque en el estudio de 'risk' Fillmore y Atkins no explotan las aplicaciones de este tipo de análisis a la traducción, Rojo y Valenzuela (1998) hemos estudiado recientemente las ventajas que este acercamiento puede aportar a la traducción de 'risk' al castellano. Comenzamos revisando las entradas de 'risk' en algunos de los principales diccionarios bilingües inglés-español/español-inglés, ${ }^{4}$ constatando una cierta confusión en las traducciones:

a) En primer lugar, no existe una distinción clara entre los términos ofrecidos en español como equivalentes de 'risk'. Los diccionarios recogen verbos como 'arriesgarse', 'exponerse' o 'atreverse', pero no está claro si dichos términos son sinónimos y pueden traducir a 'risk' en los mismos contextos o si su uso está restringido de alguna forma.

b) Hay además una cierta confusión entre el uso de la forma reflexiva (v.gr., 'arriesgarse') y la no reflexiva (v.gr., 'arriesgar') de los equivalentes.

c) Por último, la complementación sintáctica de las entradas léxicas no queda del todo clara, siendo difícil determinar los casos en los que la traducción requiere la forma ‘A + INFINITIVO' y aquellos en los que se necesita una complementación distinta.

Ante la confusión detectada en los diccionarios, la cuestión es si el esquema de 'risk' planteado por Fillmore y Atkins puede contribuir a una traducción más sistemática y funcional del término. En principio, los tres 'escenarios' o sentidos definidos por Fillmore y Atkins parecen contribuir a aclarar la confusión existente en los diccionarios bilingües:

a) En cuanto a la confusión entre los distintos equivalentes, parece que los tres 'escenarios' pueden ayudarnos a diferenciar unos términos léxicos de otros (ver Tabla 1.): mientras que 'arriesgar' puede usarse como traducción de 'risk' en cualquiera de los tres sentidos, 'exponer' sólo puede emplearse cuando hay una posesión [Po] implicada o un daño [Ma]. 'Atreverse' y 'aventurarse' se utilizan como traducciones de 'risk' cuando existe una decisión consciente por parte del protagonista.

b) Respecto al uso de los equivalentes reflexivos o no reflexivos, el esquema de 'risk' también puede servir de ayuda (ver Tabla 2.). Así, parece que la forma verbal no reflexiva sólo es posible en aquellos casos que implican el riesgo de cierta posesión [Po] mientras que la forma reflexiva puede usarse como traducción de 'risk' en cualquiera de los otros sentidos.

4. Los diccionarios bilingües consultados han sido Oxford, Collins y Larousse. 


\begin{tabular}{|c|c|l|}
\hline He risked his life & {$[\mathbf{P r}, \mathbf{P o}]$} & $\begin{array}{l}\text { Arriesgó su vida } \\
\text { Expuso su vida }\end{array}$ \\
\hline He risked falling down & {$[\mathbf{P r}, \mathbf{M a}]$} & $\begin{array}{l}\text { Se arriesgaba a caerse } \\
\text { Se exponía a una caída }\end{array}$ \\
\hline He risked climbing the mountain & {$[\mathbf{P r}, \mathbf{D e}]$} & $\begin{array}{l}\text { Se atrevió a subir la montaña. } \\
\text { Se arriesgó a subir la montaña. }\end{array}$ \\
\hline
\end{tabular}

Tabla 1. Equivalentes léxicos de RISK

c)El esquema de 'risk' también puede contribuir a aclarar la complementación sintáctica de la traducción al castellano (ver Tablas 2 y 3). En los dos casos en los que 'risk' va seguido por un SV + gerundio (nos referimos a los ejemplos que implican el mal resultado [Pr, Ma] y la decisión [Pr, De]), es posible traducir al castellano usando la estructura A + INFINITIVO, pero tan sólo en la configuración [Pr, Ma] podemos usar un SN como traducción del gerundio inglés:

\begin{tabular}{|l|l|l|}
\hline INGLÉS & SV + GER & He risked falling down \\
\hline ESPAÑOL & A + INF & Se arriesgaba A CAERSE \\
& SN & Se exponía a UNA CAIDA \\
\hline
\end{tabular}

Tabla 2. Complementación sintáctica de la traducción de RISK + SV en el escenario [Pr, Ma]

\begin{tabular}{|l|l|l|}
\hline INGLÉS & SV + GER & He risked climbing the mountain \\
\hline ESPAÑOL & $\mathbf{A}+\mathbf{I N F}$ & Se arriesgó A SUBIR la montaña \\
\hline
\end{tabular}

Tabla 3. Complementación sintáctica de la traducción de RISK + SV en el escenario [Pr, De]

En aquellos casos en los que 'risk' va seguido por un SN también es posible observar diferencias en la complementación sintáctica al traducir al español (ver Tablas 4, 5 y 6). Así, mientras que la posesión siempre se traduce por un SN y la decisión por A + INF, cuando aparece el mal resultado es posible traducir usando un SN o A + INF:

\begin{tabular}{|l|l|l|}
\hline INGLÉS & SN & He risked his life \\
\hline ESPAÑOL & SN & Arriesgó SU VIDA \\
\hline
\end{tabular}

Tabla 4. Complementación sintáctica de la traducción de RISK + SN en el escenario [Pr, Po] 


\begin{tabular}{|l|l|l|}
\hline INGLÉS & SN & He risked defeat \\
\hline ESPAÑOL & A + INF & $\begin{array}{l}\text { Se arriesgaba A SER DERROTADO } \\
\text { Se exponía a UNA DERROTA. }\end{array}$ \\
\hline
\end{tabular}

Tabla 5. Complementación sintáctica de la traducción de RISK + SN en el escenario [Pr, Ma]

\begin{tabular}{|l|l|l|}
\hline INGLÉS & NP & He risked a swim \\
\hline ESPAÑOL & A + INF & Se arriesgó A NADAR \\
\hline
\end{tabular}

Tabla 6. Complementación sintáctica de la traducción de RISK + SN en el escenario [Pr, De]

En resumen, podemos decir que los esquemas conceptuales establecidos por Fillmore y Atkins ayudan a sistematizar las elecciones léxicas de la traducción de 'risk' al castellano:
1. [Pr, Po]
ARRIESGAR / EXPONER
2. $[\mathrm{Pr}, \mathrm{Ma}]$
ARRIESGARSE / EXPONERSE
3. [Pr, De]
ARRIESGARSE / AVENTURARSE

Y también parecen contribuir a clarificar problemas de complementación sintáctica:

\begin{tabular}{|c|c|}
\hline$[$ Pr, Po $]$ & SN \\
\hline$[$ Pr, Ma $]$ & SN \\
& A + INF \\
\hline$[$ Pr, De $]$ & A + INF \\
\hline
\end{tabular}

Tabla 7. Complementación sintáctica de la traducción de RISK al español

Además, observamos que al traducir al español podría resultar útil considerar un elemento que Fillmore y Atkins habían identificado como componente del esquema de 'risk', pero que no usaron en la configuración de los distintos sentidos o 'escenarios': se trata de la causa o fuente del riesgo ( $[\mathbf{F u}]$ ), es decir, de alguien o algo que podría causar el daño. Así, los casos en los que la fuente del posible daño es un agente externo requieren ser traducidos al español usando la construcción clausal 'A QUE'. En este sentido, la interpretación del ejemplo 'He risked a punch' como posible daño o resultado negativo podría traducirse al español, por ejemplo, como 'Se arriesgaba a que le dieran un puñetazo' o incluso como 'Se arriesgaba a que se le diera un puñetazo'. 
La mayoría de las observaciones indicadas sobre la traducción de 'risk' al español son sobre todo intuitivas y aún requieren confirmación empírica; sin embargo, parecen sugerir ya cierta superioridad explicativa de la Semántica de Esquemas frente a los métodos lexicográficos tradicionales. Determinar el esquema que subyace al significado de una palabra puede contribuir a aumentar no sólo la capacidad funcional de los diccionarios, sino también la habilidad del traductor para explicar los usos que no aparecen en un diccionario.

\subsection{2. 'A Cognitive-frames Approach to Comparative Lexical Analysis' (Fillmore y Hirose 1992)}

Fillmore y Atkins $(1992,1994)$ no aplican el análisis de 'risk' a la traducción, pero esto no quiere decir que no sean conscientes de los beneficios que este tipo de análisis podría aportar a la traducción. De hecho, Fillmore y Hirose presentan un proyecto para elaborar un diccionario bilingüe japonés-inglés para la traducción automática. Intentan demostrar que un análisis basado en la Semántica de Esquemas resulta especialmente útil para la TA, en la que es necesario saber cómo se organizan los conceptos que subyacen al término ('background concepts') para poder construir las oraciones en la LM. Con este objetivo en mente, el proyecto incluye, además del método empleado en el análisis de 'risk', una fase de comparación basada en los análisis previos de cada una de las dos lenguas contrastadas. Esta fase de comparación persigue tres objetivos básicos:

- determinar la expresión gramatical de cada uno de los elementos del esquema en las dos lenguas.

- establecer los tipos de elementos necesarios en una lengua pero no en otra.

- definir las clases de restricciones que deberían incluirse en el lexicon al traducir de una lengua a otra.

El trabajo estudia tres dominios: (1) percepción; (2) espacio y (3) actos de habla. Aunque en un principio los conceptos usados en cada uno de estos dominios no parecen demasiado diferentes en inglés y en japonés, su realización gramatical presenta notables diferencias en las dos lenguas.

El proyecto de Fillmore y Hirose intenta encontrar un método sistemático para establecer comparaciones entre las dos lenguas en cada uno de los dominios semánticos establecidos. Veamos como ejemplo la comparación que ambos autores llevaron a cabo entre el verbo inglés 'see' y su correlato japonés 'mieru'. En primer lugar, se comienza identificando los elementos que componen el esquema:

\footnotetext{
Experiencer [Ex] la persona central en el esquema; el perceptor.

Discriminatum [Disc] algo que el perceptor descubre o detecta.
} 
Phenomena-as- $\quad$ [PT] el objeto detectado por la atención del

Thing perceptor. El [Disc] puede ser una parte o aspecto de éste.

Organ [Or] el órgano con el que se lleva a cabo la percepción.

Place1 [P1] la posición del perceptor.

Place2 [P2] la posición del Disc.

Distance [Dist] la distancia entre el perceptor y Disc./PT

Manner $\quad[\mathrm{M}] \quad$ la forma en la que se percibe Disc./PT.

Interpretation [Int] la apariencia del Disc./PT; su estado real puede ser distinto a la interpretación del perceptor.

Direction [Dir] la dirección hacia la que se mueve el Disc./PT.

Purpose [Ps] la razón por la que se mueve.

Instrumental [Ist] aquello con lo que el perceptor percibe el Disc./PT.

A continuación, se procede a identificar el modo en el que distintas configuraciones del esquema se llevan a cabo sintácticamente en cada una de las lenguas. Para ilustrar el procedimiento hemos seleccionado tres ejemplos en inglés y tres en japonés:

\section{Inglés:}

\begin{tabular}{|l|c|c|}
\hline Ejemplos del corpus & Elementos del esquema & Forma gramatical \\
\hline $\begin{array}{l}\text { I } \\
\text { the plane land }\end{array}$ & $\begin{array}{c}\text { Exp } \\
\text { PT (Event) }\end{array}$ & $\begin{array}{c}\text { NP } \\
\text { Acc. + Bare Inf }\end{array}$ \\
\hline $\begin{array}{l}\text { He } \\
\text { was seen } \\
\text { to enter the store }\end{array}$ & $\begin{array}{c}\text { Disc } \\
\text { PT (Event) } \\
\text { Compl }\end{array}$ & $\begin{array}{c}\text { NP } \\
\text { to-infinitive }\end{array}$ \\
\hline $\begin{array}{l}\text { We } \\
\text { saw } \\
\text { him sleeping by the roadside }\end{array}$ & $\begin{array}{c}\text { Exp } \\
\text { PT (Event) }\end{array}$ & $\begin{array}{c}\text { NP } \\
\text { Acc + Present } \\
\text { Participle Compl }\end{array}$ \\
\hline
\end{tabular}

Tabla 8. Ejemplos del análisis del inglés de Fillmore y Hirose (1992). 


\section{Japonés:}

\begin{tabular}{|l|c|c|}
\hline Ejemplos del corpus & Elementos del esquema & Forma Gramatical \\
\hline $\begin{array}{l}\text { Hidarite ni } \\
\text { suwako no hirogari ga } \\
\text { mieta toki, }\end{array}$ & $\mathrm{Pl}$ & $\mathrm{NP}+\mathrm{ni}$ \\
$\begin{array}{l}\text { Uhara wa tatte amidana kara } \\
\text { hutaribun no nimoru o } \\
\text { orosihazimeta }\end{array}$ & $\mathrm{NP}+\mathrm{ga}$ \\
\hline $\begin{array}{l}\text { 'Minasan ga soo ossyaimasu. } \\
\begin{array}{l}\text { Hontooni } \\
\text { koko kara } \\
\text { kosui ga } \\
\text { mieru to yorosii } \mathrm{n}, \\
\text { degozaimasu ga en' } \\
\text { tya o tuginagara, zyotyuu } \\
\text { ga itta. }\end{array}\end{array} \quad$ Disc. \\
\hline $\begin{array}{l}\text { Ookina ziin no yane ga } \\
\text { syoomen ni } \\
\text { mieta. }\end{array}$ & Disc. & $\mathrm{NP}+$ kara \\
\hline
\end{tabular}

Tabla 9. Ejemplos del análisis del japonés de Fillmore y Hirose (1992)

En la columna 'elementos del esquema' se especifican los elementos que participan en cada uno de los ejemplos analizados. Por ejemplo, en la primera oración de la tabla 8, 'I saw the plane land', nos encontramos con el [Ex] o perceptor ('I') y con el [PT] u objeto detectado por la atención del perceptor ( 'the plane land'); sin embargo, el primer ejemplo japonés de la tabla 9 presenta una configuración distinta de elementos del esquema, especificando la posición del perceptor [P1] y lo que el perceptor descubre o detecta [Disc]. La columna 'forma gramatical' incluye las realizaciones sintácticas de cada uno de los elementos del esquema identificados en los ejemplos. 
Una vez especificadas las distintas configuraciones y sus posibles realizaciones sintácticas en cada una de las lenguas, se procede a su comparación. Los resultados se resumen de la siguiente forma:

\begin{tabular}{|l|l|l|}
\hline Experiencer & $\begin{array}{l}|c| \\
\text { implícito (opcional); } \\
\text { si se expresa, es con el } \\
\text { caso oblicuo }\end{array}$ & explícito (obligatorio); siempre en nominativo \\
\hline Discriminatum & $\begin{array}{l}\text { opcional; si se expresa, } \\
\text { es con GA }\end{array}$ & opcional; si se expresa, es con objeto directo \\
\hline $\begin{array}{l}\text { Phenomena-as- } \\
\text { Thing }\end{array}$ & $\begin{array}{l}\text { opcional; si se expresa, } \\
\text { es con NO GA }\end{array}$ & $\begin{array}{l}\text { opcional; si se expresa, puede ser con Ac + inf } \\
\text { sin to, Compl de Inf con to, Compl de } \\
\text { Participio Presente, Compl de Cláusula } \\
\text { introducido por 'that' }\end{array}$ \\
\hline Place 1 & opcional; con KARA & opcional; con FROM \\
\hline Place 2 & opcional; con NI & opcional; con SP con varias preps \\
\hline Distance & opcional; con DE & opcional; con SP con varias preps \\
\hline Manner & $\begin{array}{l}\text { opcional; } \\
\text { con adverbiales }\end{array}$ & $\begin{array}{l}\text { opcional; } \\
\text { con adverbiales }\end{array}$ \\
\hline Organ & opcional; con NI & opcional; con wITH \\
\hline
\end{tabular}

Tabla 10. Elementos del 'frame’ y comparación de las realizaciones sintácticas en japonés y en inglés (en Fillmore y Hirose 1992).

Como conclusión de este proyecto, Fillmore y Hirose señalan una serie de ventajas que este tipo de acercamiento puede aportar al ámbito de la TA. Entre los beneficios indicados destacan los siguientes: por una parte, este tipo de análisis nos permite relacionar de manera directa los elementos que componen un esquema con sus posibles realizaciones gramaticales, acercando así semántica y sintaxis. Por otra parte, también nos permite relacionar palabras asociadas semánticamente (por ejemplo, en inglés, el esquema de VER nos permite relacionar verbos como 'see', 'look', 'watch', 'glance', 'glimpse', 'peer', 'peep', etc.), facilitando así un análisis detallado de fenómenos como la sinonimia, antonimia y, en general, de los distintos modos de expresar un concepto complejo. Es más, a diferencia de la mayoría de los sistemas computacionales, que se centran primordialmente en el estudio de dominios especializados de carácter técnico, el acercamiento propuesto por Fillmore y Hirose analiza dominios semánticos pertenecientes al lenguaje cotidiano. 
Como muestran los estudios revisados en esta sección, la Semántica de Esquemas se ha concentrado sobre todo en el estudio del significado a nivel léxico. Sin embargo, esto no quiere decir que no sea aplicable a niveles más amplios. De hecho, el propio Fillmore (1981) en un artículo titulado 'Ideal Readers and Real Readers' describe un modelo de interpretación textual basado en los postulados de la Semántica de Esquemas. A partir de aquí, cada vez son más los estudios que intentan describir el papel de los 'esquemas' en el análisis textual y discursivo. Dado que la mayoría de estos trabajos a nivel discursivo ya no pueden considerarse propiamente aplicaciones de la Semántica de Esquemas, serán revisadas en una sección aparte al final de este trabajo. Por ahora, y para concluir la sección dedicada a las aplicaciones de la Semántica de Esquemas, presentaremos la descripción de Fillmore (1981) del proceso de comprensión textual y dos aplicaciones diferentes de la noción de esquema a la traducción.

\subsection{Semántica de Esquemas e interpretación textual}

Tal y como hemos señalado, aunque hasta el momento todos los estudios revisados se han centrado en aplicaciones a nivel léxico, la Semántica de Esquemas también es aplicable a niveles más amplios. El propio Fillmore $(1975,1981)$ ha indicado la utilidad de la Semántica de Esquemas para la interpretación de textos y el análisis de discurso.

En su artículo 'Ideal Readers and Real Readers', Fillmore (1981) utiliza la Semántica de Esquemas como base de un modelo que describe el conocimiento almacenado y habilidades cognitivas que un lector necesita para interpretar correctamente un texto. En un texto escrito, las palabras sobre el papel son, utilizando una metáfora de Catherine Emmott (1997:3), como los puntos del libro de dibujo de un niño:

For a written text, the words on the page are like the dots in a gestalt figure or a child's drawing book - the connections are often obvious, but they still need to be made. To do this, readers use their knowledge stores to make inferences. (Emmott 1997, p. 3)

Los esquemas son precisamente las herramientas conceptuales que todo lector necesita para 'unir los puntos del dibujo' y construir su 'visión' ('Envisionment') del mundo del texto. El presupuesto básico que guía la aplicación de la Semántica de Esquemas al análisis textual es que ciertas formas lingüísticas se asocian en nuestra memoria con determinados esquemas, de manera que al encontrarnos con alguna de estas formas en un contexto apropiado se activa un esquema que, a su vez, nos permite acceder a otras expresiones lingüísticas asociadas con el mismo esquema. Un esquema tiene un carácter 'interactivo', puesto que es una especie de figura o esqueleto en el que no se especifican todos los detalles. En este sentido, la comprensión se considera un proceso activo en el que el que intenta comprender busca rellenar los detalles de los esquemas activados. 
La 'activación' de esquemas se puede llevar a cabo de distintas maneras: a veces son activados de forma explícita por el material linguiístico del texto; otras se derivan de inferencias apuntadas por el propio texto; incluso es posible que el lector active esquemas que no vengan indicados de forma explícita en el texto pero que son necesarios para relacionar otros esquemas y lograr así una interpretación coherente. La relación entre los esquemas puede llevarse a cabo de dos formas distintas: algunos se conectan en la memoria semántica mediante lo que Fillmore (1981:261) denomina 'vínculos de conocimiento' ( $k$-links'); se trata de conexiones basadas en nuestro conocimiento general, que se establecen independientemente de la información proporcionada por el texto. Otras conexiones vienen indicadas por el propio texto (' $T$-links'), que activa y relaciona unos esquemas con otros situándolos en el mundo que va describiendo. Estos 'vínculos textuales' se basan en un conocimiento de tipo gramatical.

En general, los esquemas activados por el intérprete de un texto estructuran tres dominios distintos pero estrechamente relacionados: el dominio del 'Contenido', que engloba las propiedades y hechos del mundo del texto; el dominio del 'Texto' y sus esquemas de estructura gramatical y textual; y el dominio del 'Género' con sus esquemas de historia de detectives, cuento popular, novela rosa y todas aquellas estructuras que organizan nuestro conocimiento sobre los distintos tipos de texto.

Veamos de qué forma todo este proceso tiene lugar en la interpretación de un segmento textual concreto. Fillmore (1981:264) demuestra el papel de estas estructuras esquemáticas en el análisis del segmento:

\section{'The carpenter was astonished $X X X X X X X X X X X X X X X X X$ '5}

Al leer este fragmento, el término 'carpenter' ('carpintero') activa en nuestras mentes el esquema de CARPINTERIA, puesto que la comprensión del sustantivo 'carpintero' está relacionada con nuestro conocimiento sobre lo que hacen los carpinteros. De esta forma, el carpintero se identifica como el trabajador en un esquema de trabajo (esto es, CARPINTERIA), que incluye, entre otras cosas, herramientas, materiales y pro-

5. Este segmento pertenece a una serie de pruebas de comprensión lectora llevadas a cabo en dos colegios de Berkeley y Oakland, California. La historia a la que dicho segmento pertenece es la siguiente:

'The carpenter was astonished that such a weird, weak-looking creature as Nasrudin was applying for a job.

'Okay, I'll give you a chance,' said the doubtful carpenter finally. 'Take this ax and chop as much lumber as you can.' At dusk Nasrudin returned.

'How many trees have you felled?' questioned the carpenter.

'All the timber in the forest,' Nasrudin replied.

Shocked, the carpenter glanced out his window. There were no trees standing on the hillside. Nasrudin had destroyed the entire forest. 'Where did you learn to chop lumber?' asked the astonished carpenter.

'In the Sahara Desert,' answered Nasrudin.

'That's ridiculous!' shrieked the carpenter. 'There aren't any trees in the desert!'

'There aren't any, now,' said Nasrudin calmly (en Fillmore 1981:248) 
ductos elaborados. Este primer esquema nos permite, además, extraer otros datos necesarios más adelante para interpretar el resto del texto, como por ejemplo la importancia de la madera como material de trabajo del carpintero: a partir de aquí podemos establecer los ' $k$-links' que nos permiten conectar al carpintero con la madera, la madera con los árboles, y los árboles con la tala y aserrado.

De forma similar a 'carpintero', 'astonished' ('sorprendido') introduce el esquema de 'Contenido' SORPRESA. En este esquema nos encontramos al menos con la persona que experimenta la emoción, el hecho que desencadena dicha emoción y las expectativas que este hecho rompe o, al menos, amenaza con romper. La conexión entre CARPINTERO y SORPRESA no se basa en nuestro conocimiento del mundo (' $K$ links') sino en información proporcionada por el texto ('T-links'): el trabajador en el esquema de CARPINTERIA es la persona que experimenta la emoción en el esquema de SORPRESA.

Además de estos esquemas de 'Contenido', Fillmore ejemplifica los esquemas 'Textuales' con el tipo de información gramatical relacionada con el verbo 'astonish' ('asombrarse'). Este verbo normalmente aparece acompañado de un complemento que describe o menciona el hecho causante de la sorpresa. Así, cuando el lector continúa leyendo, ya está preparado para integrar la información en el dominio del Contenido como la causa del asombro del carpintero. De forma más superficial, la gramática del verbo inglés también nos indica que el próximo segmento será 'at', 'to' o 'that', seguido de una descripción nominal, verbal o clausal de la causa de la sorpresa. En el caso del español, la información gramatical relativa al verbo 'asombrarse' nos indica que irá seguido de las partículas 'por', 'con' o 'de', seguidas de una descripción nominal o clausal del hecho causante del asombro.

Fillmore también ejemplifica cómo ciertos esquemas textuales pueden servir para activar un esquema de 'Género'; por ejemplo, los esquemas relacionados con el 'artículo definido' y el 'pasado simple' nos invitan a establecer una serie de expectativas sobre el tipo de texto al que pertenece el segmento. Así, nuestro conocimiento de los distintos tipos de texto nos permite identificar este relato en tercera persona, pasado simple y frases nominales 'definidas' como un tipo de NARRATIVA.

En un acercamiento de este tipo los esquemas constituyen las estructuras básicas que guían la interpretación de un texto; sin embargo, no es posible explicar el proceso de comprensión textual sin hacer referencia a ciertos principios 'pragmáticos' que nos permiten elaborar una interpretación coherente con el mínimo esfuerzo procesativo. Concretamente, Fillmore (1981) menciona el 'Principio de Parsimonia' ('Parsimony Principle'), que formula de la siguiente manera:

Don't bring more people or props into the text world than are needed to make the text cohere. (Fillmore 1981, p. 259)

Este principio intenta regular el esfuerzo de cooperación que todo lector debe realizar para lograr una interpretación coherente del texto. En este sentido, es seme- 
jante a las conocidas máximas de Grice o incluso al principio de relevancia de Sperber y Wilson (1986), puesto que se trata de estrategias que aseguran una total eficacia (a saber, máximos efectos con el mínimo esfuerzo). Así, el 'Principio de Parsimonia' de Fillmore tiene como objetivo lograr una utilización del texto que nos permita rellenar los esquemas activados de la forma más eficiente posible. Fillmore (1981:266) ilustra el funcionamiento del 'Principio de Parsimonia' con la primera oración de la historia sobre Nasrudin:

'The carpenter was astonished that such a weird, weak-looking creature as Nasrudin was applying for a job XXXXXXXXX'

Fillmore señala que sin el 'Principio de Parsimonia' sería posible imaginarnos que el carpintero es tan sólo alguien que 'observa' a Nasrudin mientras que éste espera junto a otros aspirantes para solicitar un trabajo. Sin embargo, este principio requiere usar en primer lugar los personajes de la escena para 'rellenar' los esquemas activados; por lo tanto, en esta escena de BUSQUEDA DE TRABAJO, consideraríamos a Nasrudin como el solicitante o empleado potencial y al carpintero como el entrevistador o futuro jefe.

De esta forma, los esquemas permiten a Fillmore describir el modo en el que un lector va construyendo su propia interpretación o 'visión' del mundo del texto. Es más, para el lingüista, los esquemas son las estructuras que nos ayudan a conectar esta visión que el lector va construyendo con el material léxico y gramatical del texto. En este sentido, la aplicación de la Semántica de Esquemas a la comprensión textual se convierte en un método extremadamente útil para la traducción, ámbito en el que el traductor ha de intentar reconstruir la 'visión' del lector del TO con el material léxico y gramatical de la LM. En el próximo apartado se describe una propuesta para la aplicación de la Semántica de Esquemas a la traducción.

\subsection{Semántica de Esquemas y traducción}

La relevancia de los esquemas para la traducción ha sido subrayada por un número de autores que conceden una importancia especial al proceso de comprensión (v.gr., Hietaranta 1992; Kussmaul 1995; Neubert \& Shreve 1992; Snell-Hornby 1988/1995; Wilss 1996). Sin embargo, aunque son muchos los autores que reconocen la utilidad de la noción de esquema, curiosamente tan sólo una minoría la ha utilizado en la práctica como instrumento de análisis. Uno de los pocos autores que han empleado la Semántica de Esquemas como método de análisis ha sido Paul Kussmaul (1995). Kussmaul (1995:102) concibe la traducción como la verbalización del proceso mediante el cual comprendemos un texto: '...successful translation is often nothing else but the verbalization of our comprehension'. En opinión de Kussmaul, para llevar a cabo una buena traducción, el traductor ha de basar o 'ajustar' sus decisiones al proceso de comprensión y a la función del elemento a traducir. La Semántica de 
Esquemas se convierte en un instrumento de análisis especialmente adecuado para llevar a cabo este ajuste.

Kussmaul considera apropiado mantener en traducción la diferencia inicial de Fillmore entre 'esquema' ('término lingüístico') y 'escena' ('imagen conceptual'), puesto que el término 'escena' tiene una cualidad 'plástica' o 'pictórica' que puede ayudar al traductor a comprender conceptos abstractos y a encontrar una traducción adecuada. Kussmaul (1995) ilustra la utilidad del concepto de 'escena' en la traducción del siguiente pasaje:

Man is a vertebrate, which means he has a backbone, or spine. The spine has a number of important functions in man's existence, not the least of which is the role it plays as the basis and core of the back. (Kussmaul 1995, p. 91)

La primera reacción de sus estudiantes fue traducir, de forma bastante literal, las palabras 'basis' y 'core' al alemán como 'Grundlage' ('base') y 'Kern' ('núcleo') respectivamente. Sin embargo, estas traducciones, que podrían servir en otros contextos, resultan inadecuadas en éste en concreto, puesto que 'Grundlage' sugiere una posición horizontal cuando la columna vertebral es vertical, y 'Kern' evoca interioridad mientras que la columna es central. Para estimular a sus estudiantes a producir una traducción más adecuada, Kussmaul intentó activar una 'escena' que representaba lo que como seres humanos sabían sobre sus espaldas: sobre la construcción anatómica de la espalda, con la columna vertebral bajando verticalmente desde la cabeza hasta la pelvis, y sobre lo que ocurriría si la columna se rompiera de repente. Una vez activada la escena, los estudiantes de Kussmaul produjeron una traducción más apropiada, en la que 'basis' se tradujo como 'Stütze' ('punto de apoyo') y 'core' como 'Hauptbestandteil' ('componente central o principal').

Kussmaul (1995) también analiza la relación entre los esquemas y el contexto de la historia o narración e ilustra la utilidad de los principios de 'realce' ('foregrounding') y 'supresión' ('suppression') de rasgos semánticos para la traducción de términos de significado complejo. Estos dos principios explican el hecho de que en el proceso de comprensión sólo se activan aquellos rasgos semánticos que son relevantes en un contexto dado. Este hecho ha sido demostrado en numerosos experimentos del ámbito de la psicolingüística. Un conocido ejemplo es el análisis de Barclay et al. (1974) de la palabra 'piano'. Potencialmente, la palabra 'piano' tiene bastantes características: (i.e. HEAVY, WOODEN, SOUND-PRODUCING, BEAUTIFUL, etc.) pero verbos diferentes pueden activar características distintas. Así, mientras que la oración 'The man lifted the piano' activa el rasgo HEAVY, 'The man tuned the piano' activa la característica SOUND-PRODUCING. En otras palabras, de entre todas las características contenidas en el esquema de PIANO, en cada uno de los ejemplos se realza la activada por el contexto.

El modelo de ACTIVACION DE RASGOS SEMANTICOS propuesto por Kussmaul (1995) para la traducción de términos de significado complejo (esto es, compuestos por varios rasgos distintivos) se basa en esta relación 'gestáltica' entre la activación de rasgos semánticos y el contexto de la historia o narración. Como consecuencia, el tra- 
ductor deberá mantener o realzar los rasgos relevantes y suprimir o pasar a un segundo plano los menos relevantes. Kussmaul explica este modelo utilizando un ejemplo de la traducción de Elisabeth Schnack al alemán de la historia de Saki The Open Window:

She broke off with a little shudder. It was a relief to Framton when the aunt bustled into the room with a whirl of apologies for being late in making her appearance'. (Kussmaul 1995, p. 88. Mi subrayado)

Elisabeth Schnack elige 'stürmen' ('entrar apresuradamente') como traducción de 'bustled'. Sin embargo, para Kussmaul, 'stürmen' preserva la mayoría de los rasgos semánticos presentes en el verbo inglés, concretamente los de NOISY ('haciendo ruido'), SPEEDY ('rápidamente'), EXCITED ('agitado', 'nervioso'), pero no conserva precisamente el rasgo activado en este contexto particular: BUSY ('ocupado'). Para determinar la función del pasaje y los rasgos relevantes a dicha función es necesario buscar en el proceso de comprensión del fragmento: en la historia de Saki, 'bustle' tiene como sujeto a Mrs Sappleton, una dama de mediana edad para quien las convenciones y la etiqueta son muy importantes y a quien no imaginamos entrando en una habitación de forma ruidosa, rápida o excitada, sino más bien de manera 'ocupada'. Sin embargo, este rasgo no forma parte del verbo alemán 'stürmen', que además posee ciertas connotaciones de 'fuerza' y 'velocidad', que no corresponden en absoluto al comportamiento de la dama ni a nuestro prototipo de señora de clase alta.

Por otra parte, la manera de entrar descrita por 'stürmen' tampoco encaja en la 'escena' activada en el contexto previo a este fragmento. Previamente en la historia se había activado una 'escena' en la que nos encontramos con una elegante casa donde un invitado espera a una dama mientras charla con una joven. La entrada de la dama es, pues, parte de esta 'escena' y su modo de entrar debe encajar en la situación que las palabras del narrador han evocado en la mente del lector. En este caso, la situación está impregnada del 'ideal de gentileza' que imperaba en la cultura británica en los tiempos en los que se escribió la historia, siendo difícil imaginar que acciones como 'stürmen' fueran típicas de estas situaciones. Así pues, Kussmaul señala que una traducción que se acople al proceso de comprensión sólo necesita reproducir el rasgo 'de manera ocupada'. Dado que en alemán no existe ningún término que combine los dos rasgos WALKING y BUSILY en una misma palabra, sugiere la paráfrasis 'geschäftig das Zimmer betrat' ('entró a la habitación con cara de ocupada').

El ejemplo de Kussmaul ilustra la interacción entre nuestros esquemas cognitivos previos (concretamente, el prototipo de dama y el ideal de gentileza) y los esquemas contextuales o 'escenas' formados con información procedente del texto (Mrs Sappleton como una dama preocupada por las convenciones y etiqueta y una situación de visita también gobernada por la etiqueta y el protocolo). Sin embargo, Kussmaul aún considera las palabras como conjuntos de rasgos semánticos, definición que parece estar más acorde con los postulados estructuralistas del análisis componencial ('componential analysis') que con el análisis de corte 'cognitivo' que aquí nos ocupa. 
Así pues, en los casos en los que Kussmaul habla de rasgos semánticos (por ejemplo, NOISY, SPEEDY, EXCITED), nosotros preferimos hablar de valores asignados al atributo 'manera' en el esquema de Bustle. No podemos olvidar que el interés del modelo desarrollado por Kussmaul es en última instancia pedagógico, puesto que su principal objetivo es demostrar de qué forma la Semántica de Esquemas puede ayudar a sus alumnos a mejorar la calidad de sus traducciones. En nuestra opinión, Kussmaul se aleja en cierta medida del espíritu de la Semántica de Esquemas de Fillmore al intentar combinarla con los presupuestos tradicionales del 'análisis componencial' (cf. Kussmaul 1995, p. 102); sin embargo, su modelo todavía mantiene los principios básicos del acercamiento postulado por Fillmore.

Una aplicación diferente de los esquemas en traducción aparece en el trabajo de Slobin (1997) 'Typology and rethoric: Verbs of motion in English and Spanish'. Aunque el acercamiento de Slobin no se encuadra propiamente dentro de la Semántica de Esquemas de Fillmore, la noción de 'esquema' utilizada todavía comparte ciertas características básicas con la idea de Fillmore. Por esta razón, nos parece interesante incluir una breve descripción del trabajo de Slobin.

\subsubsection{El esquema de movimiento de Talmy y traducción: Slobin (1997)}

El trabajo de Slobin (1997) constituye una de las aplicaciones más recientes de los esquemas a la traducción. Slobin basa su trabajo en el esquema de movimiento propuesto por Talmy $(1985,1991)$, quien señala que la representación conceptual de un hecho de movimiento ('motion-event frame') implica seis componentes cognitivos básicos: FIGURA ('figure'), FONDO ('ground'), TRAYECTORIA ('path'), MOVIMIENTO ('motion'), MANERA ('manner') y CAUSA ('cause'). Aunque estos elementos se consideran universales, es posible que los medios para expresar estos componentes varíen en las distintas lenguas. Concretamente, Talmy ha indicado diferencias en las expresiones de la TRAYECTORIA y la MANERA en lenguas distintas. De esta forma, Talmy distingue entre 'verb-framed languages' ('lenguas centradas en el verbo'), en las que la TRAYECTORIA se expresa a través del verbo, como en el caso del español 'entrar'; y 'satellite-framed languages' ('lenguas satélite'), en las que la TRAYECTORIA se refleja mediante una partícula, como en el caso del inglés ' $g o$ into'. Esta distinción coincide, además, con la forma en la que se expresa la MANERA: en las 'verb-framed languages', la manera de un hecho de movimiento normalmente tiene que añadirse en la forma de un adverbial mientras que en las 'satellite-framed languages' va incorporada al verbo.

Slobin señala la importancia de esta tipología en la traducción, ya que la diferencia entre los dos tipos de lenguas crea dificultades a la hora de traducir verbos de movimiento. La intención de Slobin es demostrar que las diferencias tipológicas indicadas por Talmy no sólo son interesantes desde un punto de vista sintáctico, sino que además repercuten en diferencias en los estilos narrativos de lenguas pertenecientes a tipos distintos. Con este objetivo, Slobin (1997) realiza un estudio que compara cien 
descripciones de hechos de movimiento de cinco novelas inglesas y sus traducciones al castellano con cien hechos de cinco novelas españolas y sus traducciones al inglés. En términos generales, los resultados del estudio muestran que los traductores españoles, al enfrentarse a la expresión de la MANERA, tienen que elegir entre omitir la manera y perder detalle descriptivo (como en el ej. 1) o mantener el detalle pero aumentar la complejidad sintáctica añadiendo una claúsula adverbial (como en el caso del ej. 2):

1. 'He stomped from the trim house...'

'Salió de la pulcra casa...'

2. 'She rustled out of the room...'

'Salió del cuarto, acompañada del susurro siseante de sus ropas...'

De la misma forma, en lo referente a la expresión de la TRAYECTORIA, los traductores españoles tienen que elegir entre perder detalle locativo y lograr una descripción más dinámica (como en el ej. 3) o mantener el detalle en una descripción más estática (véase el ej. 4):

3. 'He strolled across the room to the door...'

'Se dirigió a la puerta'

4. 'I climbed up the path over the cliffs towards the rest of the people.'

'Tomé el sendero que conducía al lugar donde estaba la gente'

Los resultados del trabajo de Slobin probablemente no sorprenderán a casi ningún traductor, puesto que la mayoría ya serán conscientes de las diferencias comentadas en el estudio. Sin embargo, la originalidad del estudio de Slobin reside realmente en su explicación de estas diferencias desde un punto de vista cognitivo y no meramente lingüístico. Así, Slobin señala que el inglés es más adecuado para llevar a cabo descripciones elaboradas de MANERA y TRAYECTORIA: siendo una lengua 'satellite-framed', la MANERA a menudo se incorpora al significado del verbo y la información sobre la TRAYECTORIA puede expresarse en la misma claúsula que el hecho de movimiento. Sin embargo, el español es una lengua 'verb-framed' y, por tanto, las descripciones de MANERA y TRAYECTORIA requieren construcciones adicionales que a veces resultan en un ritmo más lento de la narrativa. Por esta razón, los hablantes y traductores españoles tienden a omitir detalles de MANERA y TRAYECTORIA en favor de una descripción más dinámica.

Aunque el trabajo de Slobin no se encuadra propiamente dentro del acercamiento denominado Semántica de Esquemas, resulta obvia la relevancia para cualquier estudio que maneje las nociones de 'esquema' y 'traducción'. La siguiente sección de este trabajo se dedica también a una serie de estudios que, sin pertenecer propiamente a la Semántica de Esquemas, proponen un análisis del discurso basado en la noción de 'esquema'. 


\section{Esquemas y discurso}

En líneas generales, los lingüistas cognitivos han intentado demostrar la utilidad de las estructuras conceptuales para elaborar un modelo de procesamiento del discurso real. Uno de los principales objetivos de la LC es precisamente el de potenciar el estudio del discurso como unidad global frente a la atomización en niveles lingüísticos de las teorías estructuralistas. Incluso cuando se estudian elementos gramaticales concretos $u$ oraciones se hace desde el punto de vista de su contribución potencial al discurso. Se considera que la riqueza del significado no se revela en las formas lingüísticas aisladas sino en la dinámica del significado discursivo global.

Uno de los modelos utilizados más recientemente en LC para el análisis del discurso es la teoría de los 'espacios mentales' ('mental spaces')6 de Gilles Fauconnier (1985). En realidad, el concepto de 'espacio mental', con sus atributos de estructura flexible y dinámica, parece haber ido desplazando progresivamente al de 'esquema', frecuentemente tachado de estructura estática y poco flexible. Sin embargo, también existen trabajos que han sabido combinar la utilidad de ambos modelos. Un ejemplo interesante de esta combinación de esquemas y espacios mentales es el análisis de Richard Epstein $(1997)^{7}$ de las frases nominales definidas. Epstein comienza señalando que los hablantes utilizan el artículo definido para inducir al oyente a interpretar que el SN designa un 'rol' ('role'), y no un valor individual, dentro de un esquema ('frame'). Por ejemplo, en la oración 'The President is elected every four years', el SN 'The President' se refiere a una propiedad fija y no a un individuo específico, ya que el individuo que desempeña el rol puede variar de una ocasión a otra. Más adelante, Epstein integra esta noción de 'frame' con la de 'espacios mentales', señalando que el significado básico del artículo definido es el de indicar al oyente que el conocimiento requerido para interpretar el SN en el espacio discursivo actual está accesible en algún lugar en la configuración de espacios mentales; por lo tanto, los artículos definidos deben analizarse como marcadores formales de la intención del hablante de indicar al oyente una vía de acceso ( 'access path') al referente a través de toda la configuración dinámica de espacios. Desde este punto de vista, el artículo tiene una doble función: nos indica si construir un referente como rol o como valor individual a la vez que activa la construcción de una vía de acceso indicando qué espacios mentales y conexiones necesitamos establecer para interpretar un SN. Observemos cómo representa Epstein esta función en el siguiente ejemplo, en el que se ha traducido el análisis al español, conservando el original entre paréntesis:

[about an American television program called 'Roseanne']...last year, Roseanne's grandmother turned up at Thanksgiving dinner with a new husband

6. Dado que excede los objetivos de este trabajo, no vamos a entrar en la explicación del concepto de 'espacio mental'. Para mayor información sobre estos constructos, ver Fauconnier (1985).

7. Epstein, R. (1997) 'Roles, Frames and Definiteness'. Comunicación presentada en el $5^{\text {a }}$ Congreso Internacional de la ICLA, julio 1997, Amsterdam. 
and revealed that she had had two illegal abortions when she was young. 'Gee, I wonder who told the abortion stories at the very first Thanksgiving,' Roseanne said with a redeeming, acerbic twist...

Esquema: la cena de acción de gracias (Thanksgiving dinner)

Rol: las historias sobre aborto (abortion stories)

\section{Valores que ejemplifican el rol:}

1) las historias sobre aborto contadas en la primera cena de acción de gracias.

2) las historias sobre aborto de la abuela contadas en la última cena de acción de gracias.

$\mathbf{B}=$ base (base)

$\mathbf{R}=$ Roseanne dijo (Roseanne said)

$\mathbf{S}=$ espacio discursivo (speech space) (la primera cena de acción de gracias)

$\mathbf{L}=$ el año pasado (last year)

$\mathbf{T}=$ el esquema de ACCION DE GRACIAS (Thanksgiving frame)

$\mathbf{r}=$ rol de las historias sobre aborto (abortion stories role)

$\mathbf{v}=$ valor de las historias sobre aborto (abortion stories value)

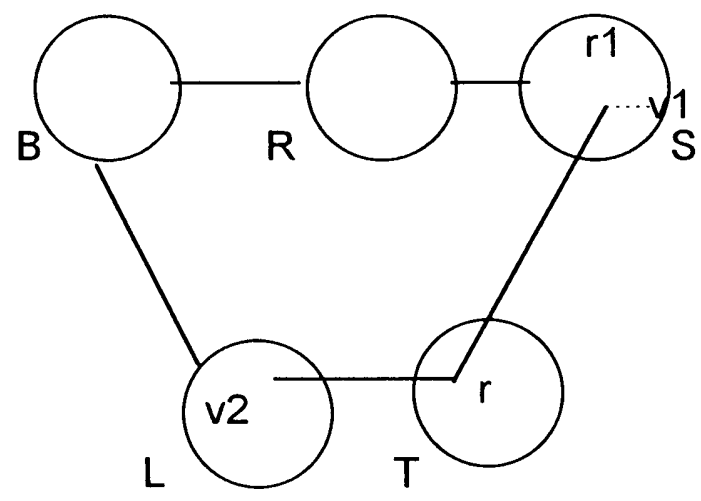

Diagrama 3.1. Diagrama para la interpretación del SN ‘the abortion stories’ (Epstein 1997)

Para explicar la función del artículo definido en este ejemplo, podemos decir que en última instancia se refiere al rol general 'las historias sobre aborto' del esquema de ACCION DE GRACIAS (T): las historias de aborto que la abuela contó durante esa cena (v2) y las contadas en la primera cena de acción de gracias (v1) son ejemplificaciones concretas de este rol. Con el diagrama, Epstein intenta representar de qué forma el conocimiento necesario para interpretar el SN 'the abortion stories' en el espacio dis- 
cursivo actual (B) está accesible en algún lugar en la configuración de espacios mentales. Así, vemos cómo la creación del espacio discursivo de la cena del año pasado (L) nos permite acceder al v2 del artículo mientras que la oración 'Roseanne said' sirve como punto de partida para la creación de otro espacio mental ( R ) que, a su vez, nos permite el acceso al v1 del artículo en el espacio discursivo de la primera cena de acción gracias ( S ). Como ya hemos mencionado, los dos valores del artículo se conectan mediante el atributo del esquema de ACCION DE GRACIAS (T).

Mediante la noción de 'esquema' y la configuración de 'espacios mentales' Epstein intenta estudiar la construcción dinámica del significado; se trata de considerar los elementos lingüísticos concretos como estímulos para dicha construcción y no como simples contenedores convencionales de significado estático. El objetivo no es sólo postular estructuras mentales que se elaboran a medida que se lee un texto sino también especificar cómo elementos lingüísticos concretos contribuyen a mantener y actualizar estas estructuras para adaptarse al desarrollo dinámico del discurso. De esta forma, los esquemas y modelos mentales se convierten en elementos de enlace entre el nivel léxico, el semántico y el discursivo, facilitando una integración de niveles que se aleja de la excesiva atomización de los análisis lingüísticos tradicionales.

El hecho de que Epstein combine 'esquemas' y 'espacios mentales' no significa que no sea posible elaborar un modelo de análisis del discurso basado únicamente en la noción de 'esquema'. Catherine Emmott (1997) constituye precisamente uno de los ejemplos más recientes de aplicación de esquemas al análisis del discurso en textos narrativos. Esta autora plantea el 'esquema' como una estructura dinámica que organiza información relativa a un texto particular. Emmott utiliza el término 'esquemas contextuales' ('contextual frames') para describir 'a mental store of information about the current context, built up from the text itself and from inferences made from the text' (Emmott 1997:121). Estos esquemas reúnen conocimiento de tipo general e información episódica del mundo de ficción, proporcionando los contextos en los que se interpretan los hechos ocurridos en la narración.

Emmott intenta demostrar que la idea de esquema aplicada al análisis de los pronombres en textos narrativos nos ayuda a identificar a los participantes de textos reales, proporcionando una explicación más completa y detallada de la forma en la que la pronominalización opera en narrativa. Veamos un ejemplo de Emmott (1997: 204-5):

Esther had forced herself to stay cheerful and strong, to go to Debby's room always with a smile. She was pregnant again and worried because of the earlier stillbirth of twin sons, but to the hospital staff, the family, and Deborah, her surface never varied, and she took pride in the strength she showed. 8 (Emmott 1997, págs. 204-5)

8. Green, H. (1985). I Never Promised You a Rose Garden. Londres: Pavanne, 33. 
Para todo lector que, como nosotros, únicamente tenga acceso a este fragmento, el pronombre 'she' podría referirse tanto a 'Debbie' como a 'Esther'. Sin embargo, los que hayan leído toda la novela de Hannah Green sabrán que se refiere al personaje de 'Esther'. Así pues, este referente no se infiere de ningún tipo de información gramatical sino de las representaciones o esquemas que el lector ha construido de ambos personajes. Estos esquemas contienen la información necesaria para la interpretación del pronombre: el hecho de que sólo Esther es una mujer adulta y por tanto capaz de estar embarazada.

Las aportaciones de los esquemas a la interpretación de los pronombres en narrativa tiene importantes repercusiones para la traducción, sobre todo si traducimos del español, que no marca el género de los pronombres posesivos, al inglés, en la que el posesivo tiene la marca de género. Consideremos por un momento el siguiente ejemplo:

¿Y qué quería decir Sheila con eso de 'pues claro que lo son'?', inquirió la Sra. Norris mientras miraba por la ventana cómo retrocedía el coche del Sr. Miles. ¿No crees, Alma, que hay algo que...?'

La Sra. Greer se lanzó sobre sus palabras con avidez.

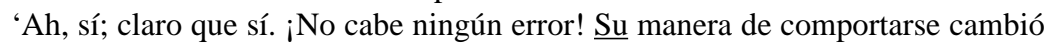
de inmediato cuando se mencionó a los Pardoe. Hasta aquel momento había parecido del todo natural.

(García Arranz 1990, p. 17. Mi subrayado)

Aunque García Arranz traduce el 'su' como 'her' al inglés, el extracto no proporciona la información necesaria para determinar si la señora Greer se está refiriendo a Sheila o al señor Miles. El traductor necesita recurrir a su esquematización de situaciones anteriores de la obra para identificar qué personaje cambió su manera de comportarse al mencionar a los Pardoe. La identificación del personaje es fundamental para una correcta traducción al inglés, lengua en la que hemos de elegir entre el femenino 'her' y el masculino 'his'.

Tanto el estudio de Epstein (1997) como el de Emmott (1997) intentan demostrar la utilidad de los esquemas en el análisis de ciertas formas lingüísticas con un particular valor discursivo, como es el caso del artículo 'the' y los pronombres. Se trata, por tanto, de estudios que abordan el análisis del discurso desde una perspectiva predominantemente lingüística. Sin embargo, en la aplicación de los esquemas al análisis del discurso es posible definir otra línea de investigación de corte más 'literario'. Dicha línea de investigación está constituida por una serie de estudios (v.gr., Cook 1990, 1994; Semino 1996) que defienden la utilidad de los esquemas para estudiar el proceso de comprensión de textos literarios, puesto que nos permiten explicar la interacción entre los textos y el conocimiento del lector. Desde este punto de vista, la teoría de los esquemas se ha considerado un método útil para describir cómo se construyen los mundos textuales en el procesamiento discursivo.

En líneas generales, los estudios que aplican los esquemas a la literatura resaltan como cualidad de los textos literarios su tendencia a modificar los esquemas previos 
del lector. Desde este punto de vista cognitivo, la 'cualidad literaria' de un texto ya no se considera una propiedad formal de un determinado tipo de textos sino más bien una propiedad de un cierto tipo de procesamiento textual que tiende a modificar los esquemas del lector. Los textos literarios ya no se definen simplemente por sus características estéticas sino por su potencial para causar un cambio en los esquemas del que los interpreta. En este sentido, Cook (1990) afirma que el discurso literario desempeña la función cultural de crear las condiciones necesarias para modificar los esquemas del lector.

En opinión de Cook, la literatura proporciona a los lectores la oportunidad de reorganizar sus esquemas sin incurrir en sanciones sociales o prácticas inoportunas. De esta forma, el discurso literario se distingue del resto porque las desviaciones que aparecen en el texto cuestionan los esquemas del lector ('schema disruption'), y resultan en un cambio ('schema refreshment'). A diferencia del discurso literario, Cook indica que el publicitario se caracteriza simplemente por confirmar esquemas ya existentes ('schema reinforcing') o proporcionar información nueva para ser incorporada dentro de estos esquemas ( 'schema adding').

Aunque este tipo de acercamiento se centra en la interacción entre los lectores y los textos, la mayoría de los trabajos han descuidado la interrelación entre las formas lingüísticas concretas y los esquemas. Así, aún es necesario llevar a cabo más estudios que analicen el papel de las elecciones y expresiones lingüísticas concretas en la activación y modificación potencial de los esquemas. El trabajo de Elena Semino (1995) apunta en esta dirección, intentando demostrar que la activación de determinados esquemas ayuda al lector de textos poéticos a establecer la coherencia del poema, llevando a cabo las conexiones necesarias entre las acciones, personajes y objetos de éste. Así por ejemplo, Semino argumenta que la interpretación del poema 'A Pillowed Head'9 de Seamus Heaney depende fundamentalmente de la activación del esquema de ALUMBRAMIENTO ('CHILDBIRTH'), al cual se accede mediante las repetidas referencias a sus componentes; entre dichas referencias destacan: 'the pangs' (verso 8), 'the

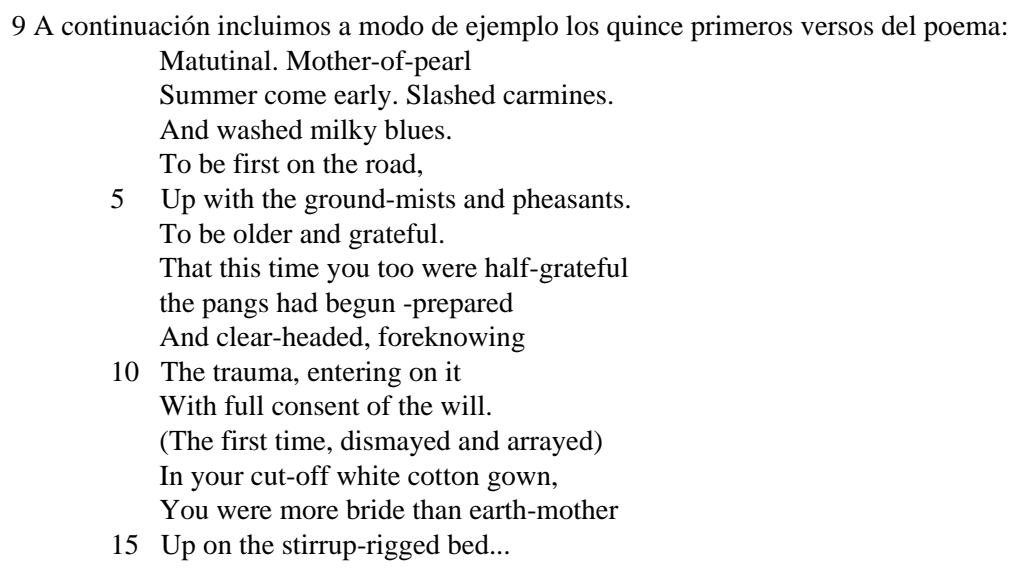

10 The trauma, entering on it With full consent of the will. (The first time, dismayed and arrayed) In your cut-off white cotton gown, You were more bride than earth-mother

15 Up on the stirrup-rigged bed... 
trauma' (verso 10), 'your cut-off white cotton gown' (verso 13), 'earth-mother' (verso 14), 'the stirrup-rigged bed' (verso 15), etc.

A lo largo de todo este trabajo, hemos intentado señalar algunas de las ventajas que un análisis basado en el concepto de 'esquema' presenta para el estudio lexicográfico, el análisis del discurso y la traducción. Sin embargo, sería extremadamente pretencioso por nuestra parte negar la existencia de ciertos problemas relativos a los esquemas. Por esta razón, creemos conveniente mencionar las principales dificultades de este tipo de análisis.

\section{Problemas relacionados con los 'esquemas'}

En líneas generales, los problemas relacionados con los esquemas se pueden reducir a dificultades de dos tipos:

a) Las relativas a la formalización precisa de las estructuras. Han sido sobre todo los expertos en IA los que han señalado las complicaciones en la formalización semántica de estas estructuras. Para ser 'implementables' en un programa de ordenador, los esquemas deben definirse con una precisión casi 'matemática'. Sin embargo, esta exactitud resulta difícil de alcanzar al tratarse de estructuras de organización prototípica, que permiten codificar tanto los ejemplos típicos como las excepciones de una categoría. Las complicaciones aumentan a medida que se intenta formalizar ejemplos más complejos, como es el caso de determinados fragmentos discursivos. A continuación, presentamos un ejemplo en el que Barsalou (1992b:210-211) ha utilizado los esquemas para representar el siguiente fragmento discursivo:

Rick, feeling festive, rented a house near a tropical reef. He had recently built a house worth three million dollars. Rick had visited the reef long ago. The fish had been beautiful, and many divers were present

Dado que con el ejemplo de Barsalou tan sólo pretendemos ilustrar las dificultades para formalizar un análisis del discurso basado en los esquemas, no entraremos en una descripción detallada del diagrama. A modo de breve explicación, señalaremos que este modelo intenta representar cómo las proposiciones de un texto se integran para construir lo que Barsalou denomina 'un modelo coherente de referencia' (' $a$ coherent referent model'). Así, la primera oración ('Rick, feeling festive, rented a house near a tropical reef') activa los esquemas RICK, RENT, HOUSE y REEF. El número en cada esquema indica la oración en la que se menciona por primera vez (por ejemplo, RICK $^{1}$ señala que aparece por primera vez en la oración 1 del texto). A medida que el texto avanza, las proposiciones nuevas se integran con las anteriores determinando las expresiones lingüísticas que se refieren a las proposiciones de oraciones previas (es decir, mediante 'anáfora'). Así, cuando Rick aparece en la oración 2, se asume que se refiere al mencionado en la oración 1 y, por lo tanto, se representa como el agente tanto de la proposición de RENT como de la proposición de BUILD. Sin embargo, hay 


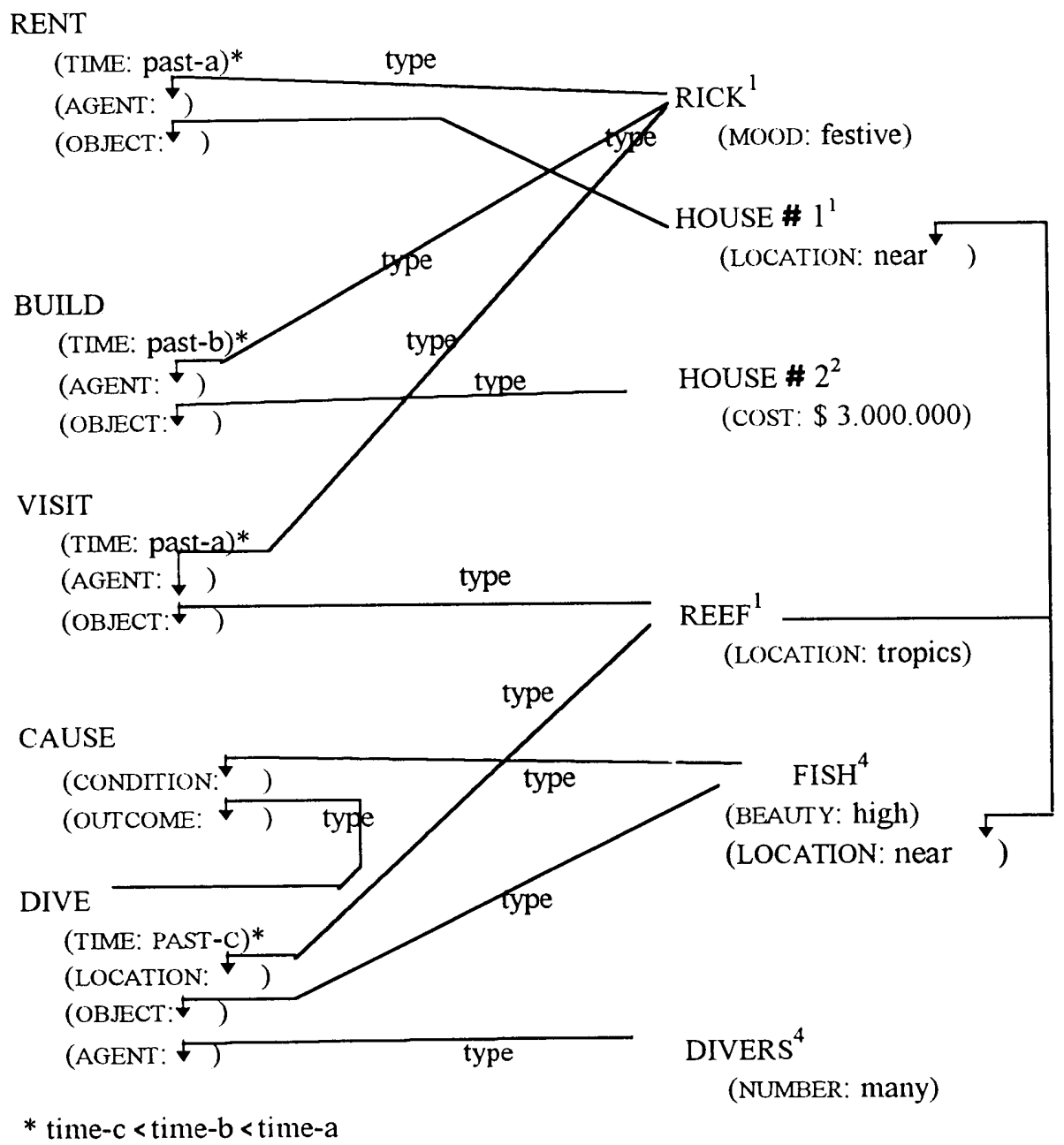

Diagrama 4.1. Ejemplo de uso de 'frames' para representar el discurso (Barsalou 1992b, p. 211)

que tener en cuenta que la repetición no siempre indica 'co-referencia' ('co-referen$c e$ '). Así, el término 'house' de la oración 1 y el de la oración 2 representan distintos ejemplares de la categoría; por lo tanto, HOUSE \# 1 se representa como objeto de RENT mientras que HOUSE \# 2 se sitúa como objeto de BUILD.

Como es posible apreciar a simple vista, incluso en un fragmento tan breve como éste la complejidad de la representación refleja los problemas para formalizar un análisis de este tipo. El mismo Barsalou admite la complejidad del modelo de la siguiente forma: 
As we have seen from this example, integrating discourse propositions is extremely complicated: Sometimes a repeated word indicates co-reference and sometimes not. Usually a new word does not indicate co-reference, but sometimes it does. Comprehension often requires the inferring of new referents that the text does not mention explicitly, as well as implicit relations between propositions. (Barsalou 1992b, p. 212)

Otro tipo de dificultades para la formalización de los esquemas provienen de la naturaleza a menudo abstracta de las entidades y experiencias que conceptualizan. Por ejemplo, para describir el significado de palabras aparentemente tan simples como 'delante', 'atrás', 'sonreír', 'comida' o 'charla', necesitamos referirnos, entre otras cosas, a factores tan difícilmente formalizables como las interacciones en el espacio con el entorno que nos rodea, experiencias universales como la alegría o la tristeza y universales culturales relativos a la alimentación y habla.

b) El otro tipo de problemas se refieren a las dificultades en la delimitación de los esquemas. Por una parte, se plantean problemas relativos a la definición de los elementos o atributos que componen un esquema. En la mayoría de los casos, no está claro cuántos atributos son necesarios para definir un esquema de forma completa: por ejemplo, para definir el esquema AMIGO podríamos utilizar atributos como 'nombre', 'edad', 'raza' y seguir añadiendo atributos casi indefinidamente.

Por otra parte, también se plantean problemas a la hora de delimitar unos esquemas de otros. Frente al ideal de esquemas perfectamente acotables, a menudo nos encontramos esquemas con un significado demasiado cercano o incluso procesos complejos que constituyen una especie de continuum difícil de delimitar y que nos permiten clasificar un mismo término en distintos esquemas según sus componentes (p. ej., 'injertar' podría clasificarse en cualquiera de los esquemas de 'cortar', 'separar', 'trasladar' o 'unir').

En general, la noción de 'esquema' parece ser todavía un tanto vaga y quizás demasiado imprecisa para resultar de utilidad práctica. Por esta razón, es necesario que todo estudio que intente aplicar la noción de esquema defina claramente su interpretación del término. En líneas generales, los problemas mencionados en relación al uso de los esquemas son consecuencia de intentar 'encasillar' la realidad en estructuras de carácter general y hasta cierto punto idealizado que no se corresponden con exactitud al mundo que nos rodea. Por lo tanto, es necesario mantener siempre un cierto margen de flexibilidad en la aplicación del esquema, puesto que a menudo resulta imposible captar todas y cada una de las particularidades de los casos concretos.

\section{Una última y breve reflexión}

No quisiéramos que tras revisar las dificultades planteadas por los esquemas, los lectores de este trabajo encontraran más problemas en el uso de estas estructuras que beneficios. No podemos olvidar que la Semántica de Esquemas es todavía una teoría en evolución y que es preciso continuar trabajando en su elaboración y desarrollo. Pero pese a sus limitaciones, es imposible negar que sus presupuestos apelan a nuestro 
sentido común y habilidades cognitivas básicas. A nivel teórico, nos proporciona una visión de la lengua totalmente coherente con los más recientes descubrimientos sobre la cognición humana. A nivel práctico, las nociones de ‘esquema' y 'prototipo' nos permiten sistematizar y organizar nuestro conocimiento cultural e integrarlo con información de tipo lingüístico.

Para los lexicógrafos, la Semántica de Esquemas se convierte en una útil herramienta de análisis que les permite relacionar de manera directa los elementos que componen un esquema con sus posibles realizaciones gramaticales, acercando así semántica y sintaxis. Para críticos literarios, analistas del discurso y traductores, un modelo basado en la noción de 'esquema' resulta interesante puesto que permite explicar fenómenos lingüísticos más allá del nivel oracional. Leer o interpretar un texto implica establecer una relación entre la mente del lector o intérprete y el texto en sí. Por esta razón, para un analista del discurso es importante tener en cuenta las habilidades cognitivas que el texto demanda del lector. En este sentido, Emmott (1997) ha argumentado que los esquemas son entidades dinámicas que nos permiten explicar estas demandas, tanto en lo referente al establecimiento de inferencias como en lo relativo al almacenamiento mental de información. Desde una perspectiva cognitiva de este tipo, el análisis llevado a cabo puede contribuir a comprender los mecanismos mentales que subyacen a la interpretación de los elementos culturales en el discurso. Más allá del nivel léxico y oracional, los esquemas nos ayudan a entender la interacción entre la información textual y nuestros almacenes de información cultural.

\section{Bibliografía}

ATKINS, B.T.S. (1994) 'Analyzing the verbs of seeing: a frame semantics approach to corpus lexicography'. B.L.S 21: 42-56.

Barsalou, L.W. (1992) Cognitive Psychology. An Overview for Cognitive Scientists. Hillsdale, NJ: Lawrence Erlbaum Associates.

Cook, G. (1990) A Theory of Discourse Deviation: the Application of Schema Theory to the Analysis of Literary Discourse. Tesis Doctoral. Universidad de Leeds.

Cook, G. (1994) Discourse and Literature: The Interplay of Form and Mind. Oxford: Oxford University Press.

Embleton, S. (1991) 'Names and Their Substitutes. Onomastic Observations on Astérix and Its Translations'. Target 3 (2): 175-206.

Eммотт, C. (1997) Narrative Comprehension. A Discourse Perspective. Oxford: Oxford University Press.

Epstein, R. (1977) 'Roles, Frames and Definiteness'. Comunicación presentada en el $5^{\text {a }}$ Congreso Internacional de la ICLA, Amsterdam, julio 14-19, 1997.

FAuconnier, G. (1985) Mental Spaces. Cambridge Mass.: MIT Press.

Fillmore, CH. J. (1975) 'An Alternative to Checklist Theories of Meaning'. BLS 1: 123-131. 
Fillmore, CH. J. (1981) 'Ideal Readers and Real Readers', Georgetown University Round Table on Language and Linguistics, D. Tannen (ed.), 248-70.

Fillmore, Ch. J. y AtKins, B.T.S. (1992) 'Toward a Frame-based Lexicon: the Semantics of RISK and its Neighbors', en E. Kittay y A. Lehrer (eds.) Frames, fields and contrasts: New essays in semantic and lexical organization. Hillsdale, NJ: Lawrence Erlbaum Associates, 75-102.

Fillmore, CH. J. y Atkins, B.T.S. (1994) 'Starting Where Dictionaries Stop: the Challenge of Corpus Lexicography', en Atkins, B.T.S \& A. Zampolli (eds.) Computational Approaches to the Lexicon. Oxford: Oxford University Press.

FrameNet Project. International Computer Science Institute. UCB Department of Linguistics. http://www.icsi.berkeley.edu/ framenet Documento impreso el día 11 de diciembre de 1997, 1-6.

Garcia Arranz, A. (1990) Traducciones de español a inglés. Madrid: Sociedad General Española de Librería, S.A.

Hietaranta, P. (1993) 'Framing Languages: Towards a Frame-Theoretical View of Translation', en Y. Gambier y J. Tommola (eds.) Translation and Knowledge. IV Scandinavian Symposium on Translation Theory, Turku: University of Turku, 115-127.

Kussmaul, P. (1995) Training the Translator. Amsterdam: John Benjamins Publishing.

Neubert, A. y Shreve, G. M. (1992) Translation as Text. Kent: The Kent State University Press.

Rojo, A.M. y Valenzuela, J. (1998) 'Frame Semantics and Lexical Translation. The Risk Frame and its Translation'. Babel 44:128-38.

Rosch, E. (1973) 'Natural Categories'. Cognitive Psychology 4: 328-350.

Rosch, E. (1978) 'Principles of Categorization', en E. Rosch y B.B. Lloyd (eds.) Cognition and Categorization. Hillsdale. N.J.: Lawrence Erlbaum Associates, 27-48.

Rosch, E. Y Mervis, C. (1975) 'Family Resemblances: Studies in the Internal Structure of Categories'. Cognitive Psychology 7: 573-605.

Semino, E. (1995) 'Schema Theory and the Analysis of Text Worlds in Poetry'. Language and Literature 4 (2): 79-108.

Slobin, D.I. (1997) 'Typology and Rhetoric: Verbs of Motion in English and Spanish', en M. Shibatani y S. A. Thompson (eds.) Grammatical Constructions: Their Form and Meaning. Oxford: Oxford University Press.

Snell-Hornby, M. (1988/1995) Translation Studies. An Integrated Approach. Amsterdam: Benjamins.

SPERBER, D. Y WILSON, D. (1995) Relevance: communication and cognition. Oxford: Blackwell.

Vega DE, M. (1984) Introducción a la Psicología Cognitiva. Madrid: Alianza.

WiLss, W. (1996) Knowledge and Skills in Translator Behavior. Amsterdam/ Philadelphia: John Benjamins. 